EPiC Series in Computing
Volume 62, 2019, Pages 101-106
SUMO User Conference 2019

\title{
Pedestrian-Friendly Traffic Signal Control Using Eclipse SUMO
}

\author{
Gorkem Akyol $^{1}$, Mehmet Ali Silgu ${ }^{1,2^{*}}$ and Hilmi Berk Celikoglu ${ }^{1}$ \\ ${ }^{1}$ Technical University of Istanbul, Faculty of Civil Engineering, ITS Research Lab., Ayazağa \\ Campus, Istanbul, Turkey \\ ${ }^{2}$ Bartin University, Faculty of Engineering, Department of Civil Engineering, Bartin, Turkey \\ \{akyolgo, msilgu, celikoglu\}@itu.edu.tr, masilguebartin.edu.tr
}

\begin{abstract}
The center of the Kadıköy area in Istanbul is extremely crowded due to overlap of the terminals for the subway and the marine transit lines. When the Kadiköy-Kartal subway's terminal is added in the middle of the Kadıköy in 2013 without the analysis on crowd dynamics, vehicular and pedestrian traffic have become much more complicated to be efficiently managed. When the pedestrians have a crossing gap, most of them make the decision of crossing without considering the signal phase. Likewise, when it is a pedestrian clearance phase, there can be situations where all the pedestrians cannot cross the street because of high density and insufficient green time. We therefore propose an adaptive traffic control system considering the traffic flows of road vehicles and pedestrians with field data. We have utilized the Eclipse SUMO micro-simulator in conjunction with TraCI for modeling the case. Comparison of fixed time and adaptive signal controllers is provided. Simulations have shown that, reductions in the delay time for both vehicles and pedestrians are achieved by using adaptive signal controllers.
\end{abstract}

\section{Introduction}

This paper focuses on the optimization of two traffic lights in the Kadıköy area -one of the central business districts in the polycentric form of Istanbul city- that is used by excessive amount of pedestrians. If such a high density pedestrian crowd is not considered carefully, several problems are likely to exist regarding the increased delay time for each pedestrian and total time spent overall in the system. We therefore propose an adaptive traffic control system considering the traffic flows of road vehicles and pedestrians field data using Eclipse Simulation of Urban Mobility (SUMO) micro-

\footnotetext{
${ }^{*}$ Corresponding Author: Mehmet Ali Silgu
} 
simulator in conjunction with TraCI. Adaptive traffic signal control is defined in Signal Timing Manual (Koonce et al., 2008) as a signal control concept where vehicular traffic in a network is detected with different types of detectors to make signal adjustments for compensating varying demand of traffic. The detectors can be placed at upstream and/or downstream of the intersections to predict the current traffic situation.

The paper is organized as follows: Section II gives information on existing literature; In Section III, modeling progress of the proposed system with Eclipse SUMO and characteristics of the case area are explained; Section IV provides the discussion of the simulation results for both pedestrians and vehicles; Section V covers the conclusion and future research.

\section{Literature Review}

Existing literature about the pedestrian green time optimization is introduced in this chapter. Helbing et al. (2005) evaluated the crowd dynamics with several tests and found several patterns such as lane formation, stripe formation and shockwaves in dense crowds. They conducted tests on different areas like corridors, bottleneck areas and intersections to examine the Self-Organization phenomena.

Ding et al. (2006) created a pressure-based model to mimic the traffic control agencies' (police officers, firefighters etc.) decision-making behavior. By this model, waiting traffic units create a pressure at the traffic control agency (TCA) and when this pressure reaches a critical value, TCA terminates the current phase and switches to another.

Zade and Dandekar (2012) applied fuzzy interference system (FIS) to create a traffic responsive signal control. They created a fuzzy system that consists of two important characteristics: collecting data from on-going traffic at the intersection and adaptive traffic signals that respond to the data. Their fuzzy logic for traffic density ranges from very small $(0-40 \mathrm{veh} / \mathrm{km})$ to extremely large (80-100 $\mathrm{veh} / \mathrm{km}$ ) of density.

Ma et al. (2015) investigated the different phase patterns (exclusive phase patterns (EPP) and two way crossing (TWC) for increasing both efficiency and safety. In their research, they measured the operational efficiency with different delay types and safety efficiency considering two parameters. Operational efficiency parameters are; signal delays, conflict delays and detour delays whereas safety parameters are the number of pedestrian-vehicle conflict and pedestrians' compliance rate of rules. Then, they created a cost function that converts these factors into monetary values to select phase patterns and optimize signal timings.

Oskarbski et al. (2016) conducted a study at one intersection in Poland for increasing pedestrian safety by reducing severe incidents that occur between vehicles and pedestrians having data obtained by video camera mounted for scanning the intersection at a height of 4.5 meters. For data analysis, age and gender of the pedestrians are used as parameters. They calculated crossing speed of pedestrians for different scenarios and compared the results having the simulation software VISSIM.

Considering the relevant existing studies, the novel contribution of the present study is that it takes pedestrians and vehicles into account to assign cycle times and phase durations in an adaptive fashion. In addition, this study is conducted using real data from a very crowded district in Istanbul.

\section{Modelling and Simulation}

In the following subsections, the methods used to model and analyze the real case is explained in part. 


\subsection{Case Study Area}

Case area can be seen in Figure 1. Orange line is indicating the tram's route in the area. Red arrows are showing the locations of two consecutive traffic lights, throughout of which the extensive pedestrian traffic flows. Black arrows are the location of ferries that have a 1500 people capacity. Blue arrows are showing the subway entrance and exit points. Yellow polygon is the center of Kadikoy where one can find shopping malls, dining restaurants, pubs and historical places.

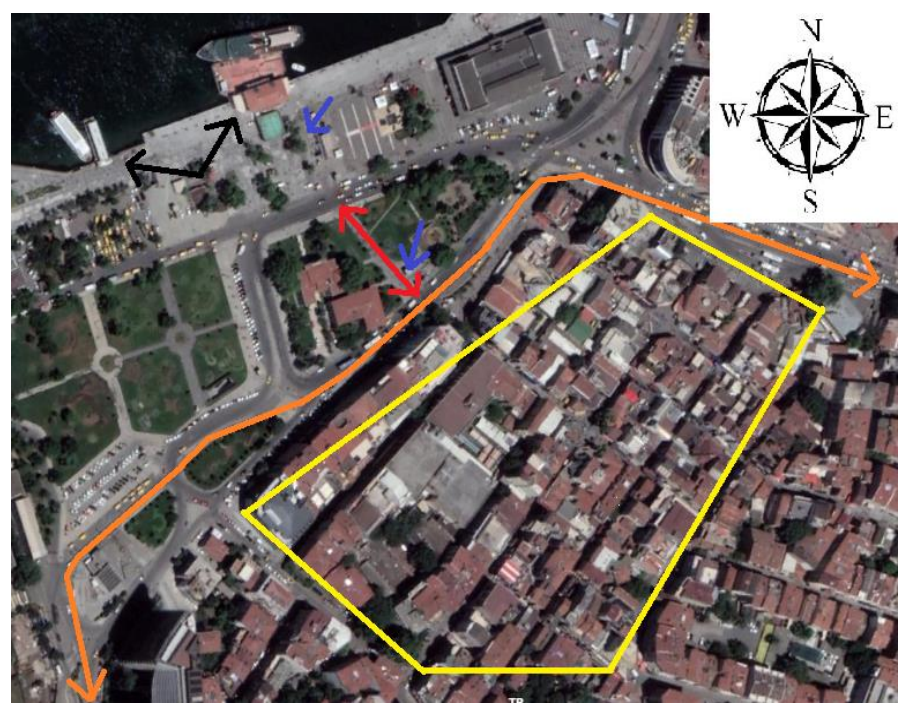

Figure 1. Network of the case area.

In our case, two pedestrian crosswalks are considered for evaluation. Dimensions of these crosswalks are given in Table I. The intersection located at the northern bound of the red arrow in Fig. 1 is named "Ferry" intersection while the intersection located at the southern bound of the red arrow in Fig. 1 is named "City Center" intersection. Two intersections at the bounds of red arrow are oneway for vehicular traffic. The "Ferry" intersection accommodates three lanes for vehicular traffic whereas "City Center" intersection contains two lanes to serve vehicles and the tram line as well.

Table I. Geometric properties of the intersections.

\begin{tabular}{lll}
\hline \multirow{2}{*}{ Location } & \multicolumn{2}{c}{ Intersection Geometry $(\mathbf{m})$} \\
\cline { 2 - 3 } & Length & Width \\
\hline Ferry & 15.6 & 8.3 \\
\hline City Center & 12.3 & 5.2 \\
\hline
\end{tabular}

Features of data are retrieved from camera recordings that are captured from two locations surrounding the case area. Observation of the two intersections in the case area is done on the 26th of January, 2019. The recording process is conducted in both morning peak hours (07:00-10:00) and evening peak hours (16:00-19:00). Data aggregation is processed manually.

In the real-case, traffic lights have 105 seconds cycle time. Four phases are included in this cycle, which are 75 seconds for vehicles to pass the intersection, 5 seconds of all-red phase for intersection clearance, 20 seconds for pedestrian green time and 5 seconds of all-red phase for clearance again. 


\subsection{Simulation Environment}

Microsimulation software Eclipse SUMO uses two different pedestrian dynamics models to simulate the behavior of pedestrians (Erdmann \& Krajzewicz, 2015) and (Lopez et al., 2018):

1. Non-Interacting Model

2. Striping Model

First model is very simple and used when the pedestrians interact with each other and other traffic units are not important. Pedestrians move with a constant speed and they jump across through intersections. By using this model, one can achieve low computational times.

Striping model has more detailed properties in terms of pedestrian dynamics. Firstly, with this model, interactions between pedestrians and vehicles can be observed and more valid results can be obtained for traffic management solutions. To achieve collision avoidance, this model divides the lateral width of a lane into discrete bars with fixed width. These bars are similar to lanes of a multilane road. For that reason, collision avoidance is achieved by keeping a sufficient distance within the same bar. If a pedestrian comes near to another pedestrian, it moves in the y-direction (lateral movement) and in the $\mathrm{x}$-direction (longitudinal) to change its stripe to avoid collision.

For simulation, network is imported from Open Street Map data base. Case area has 50 nodes and 54 edges with 2 consecutive traffic lights.

For creating sidewalks, edges on the imported network are converted into the sidewalks and walking areas. 12 edges are defined for pedestrians to get through their destination (ferry, bus, metro, sightseeing etc.). Pedestrian flows are created with embedded Python code.

For simulating pedestrian motion in the simulation environment, three different points in the case area are used. Firstly, 15 minutes of cyclic inputs for the ferry passengers arriving from the European side of the Istanbul is modeled. Secondly, 6 minutes of cyclic inputs for the subway passengers is modeled. Lastly, pedestrians that are counted from video recordings are modeled in the simulation environment.

Vehicle flows are created with identifying vehicle types firstly, (id, acceleration and deceleration rates, length of the vehicles etc.) and then with the flow property of Eclipse SUMO routes. 5000 vehicles are assumed to enter the simulation within 3600 seconds and 1800 pedestrians are assumed to move in the system. Simulation lasts for 9071 seconds to let all the vehicles clear the simulation.

Simulations are conducted 10 times in order to reflect stochastic nature of traffic demand.

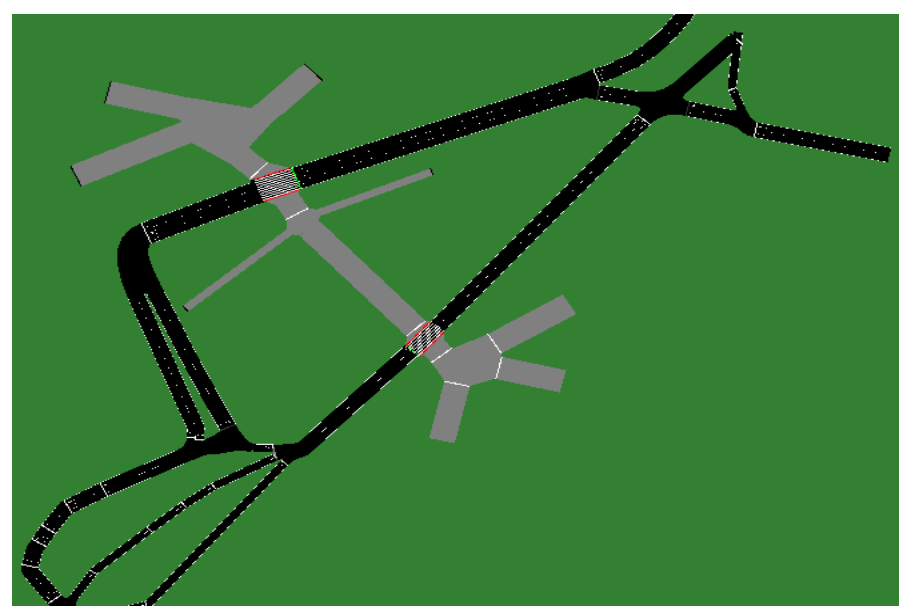

Figure 2. Simulation environment created with Eclipse SUMO 


\subsection{Traffic Lights and Programs}

There are several ways to implement traffic lights in Eclipse SUMO environment. Traffic lights and programs at intersections are generated using NETCONVERT and NETEDIT at the intersections considered. Eclipse SUMO/SUMO-GUI is used to define different signal programs for different traffic lights. Lastly, NETEDIT is used to edit traffic light plans visually.

In our case, our network file and route file are then merged into the configuration file. Additional files are needed to be added in the configuration file in order to implement traffic light program. To adapt TraCI in our simulation, embedded Python code named runner.py is altered in our case. For this purpose, we changed minimum green time for vehicles to 60 seconds from 15 seconds to achieve less delay time for vehicles.

\section{Results and Discussion}

With the results obtained, one can see the total time loss for both pedestrians and vehicles. Time loss is defined in (SUMO, 2019) as the time lost due to driving below the desired speed. Desired speed includes the speedFactor attribute for each vehicle. SpeedFactor attribute is multiplied with the speed limit to determine desired driving speed. In our case, this attribute is set to its default value, which is 1 . Graphical interpretation of the results are given in Figure 2. It is shown that, by using adaptive signal controllers, total time loss in the system can be reduced for both pedestrians and vehicles. Fixed time solutions may offer lower computational time and are easy to be implemented. Because of the usage of historical data, fixed-time solutions cannot compensate the changing demand of traffic. Adaptive signal controllers can be used for reducing total time spent in the system, which leads less emissions, and fuel consumption, though their implementation is not that simple compared to the fixed-time signal controllers. They need centralized control stations and state-of-the-art detection systems.
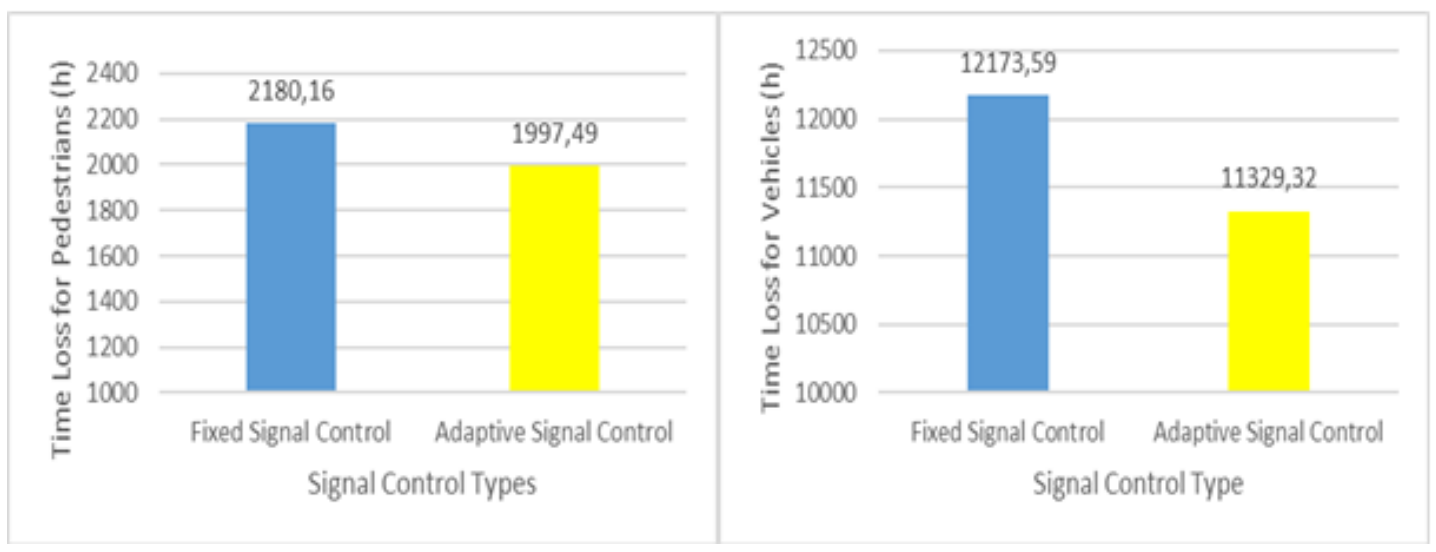

Figure 3. Time loss for types of signal control 


\section{Conclusions and Future Research}

This paper provides recent literature on the problem handled and implementation of adaptive signal controllers by using microsimulation software Eclipse SUMO. In conclusion, using adaptive signal controllers can increase the efficiency of intersections by reducing total time loss of vehicles and pedestrians by $7 \%$ and $9 \%$ respectively. For the future research, addition of the tram line and its effects to analyses is to our interest. The real time control model can further be calibrated with more detailed subway and ferry e-ticket data.

\section{References}

Ding, N., He, Q., Wu, \& C., Fetzer, J. (2015). Modeling Traffic Control Agency Decision Behavior for Multimodal Manual Signal Control under Event Occurrences. IEEE Transactions on Intelligent Transportation Systems, 16(5), 2467-2478., doi:10.1109/tits.2015.2409174

Erdmann, J., \& Krajzewicz, D. (2015). Modelling pedestrian dynamics in SUMO.

Helbing D., Buzna L., Johansson A., \& Werner T. (2005). Self-organized Pedestrian Crowd Dynamics: Experiments, Simulations and Design Solutions. Transportation Science 39(1), 124.

Koonce, P., Rodegerts, L., Lee, K., Quayle, S., Beaird, S., Braud, C., et al. (2008). Traffic Signal Timing Manual. Report No. FHWA-HOP-08-024. Federal Highway Administration, Washington, D.C.

Lopez, P.A., Behrisch, M., Bieker-walz, L., Erdmann, J., Flötteröd, Y., Hilbrich, R., et al. (2018). Microscopic Traffic Simulation using SUMO. 21st International Conference on Intelligent Transportation Systems (ITSC), Maui, HI, 2575-2582.

Ma, W., Liao, D., Liu, Y., \& Lo, H. K. (2015). Optimization of pedestrian phase patterns and signal timings for isolated intersection. Transportation Research Part C: Emerging Technologies, 58, 502-514., doi:10.1016/j.trc.2014.08.023

Oskarbski, J., Guminska, L., Miszewski, M., \& Oskarbska, I. (2016). Analysis of Signalized Intersections in the Context of Pedestrian Traffic. Transportation Research Procedia, 14, 2138-2147., doi:10.1016/j.trpro.2016.05.229

SUMO Wiki Webpage - Information for Simulation/Pedestrians.

Zade, A., \& Dandekar, D.R. (2012). Simulation of Adaptive Traffic Signal Controller in MATLAB Simulink Based On Fuzzy Inference System. 